\title{
DE L'ALLEMAGNE : UN LIVRE SUR LA FRANCE
}

\author{
Winfried Wehle
}

Presses Universitaires de France | Revue d'histoire littéraire de la France

2014/2 - Vol. 114

pages 318 à 334

\section{ISSN 0035-2411}

Article disponible en ligne à l'adresse:

http://www.cairn.info/revue-d-histoire-litteraire-de-la-france-2014-2-page-318.htm

Pour citer cet article :

Wehle Winfried, « De l'Allemagne : un livre sur la France »,

Revue d'histoire littéraire de la France, 2014/2 Vol. 114, p. 318-334. DOI : 10.3917/rhlf.142.0318

Distribution électronique Cairn.info pour Presses Universitaires de France.

(c) Presses Universitaires de France. Tous droits réservés pour tous pays.

La reproduction ou représentation de cet article, notamment par photocopie, n'est autorisée que dans les limites des conditions générales d'utilisation du site ou, le cas échéant, des conditions générales de la licence souscrite par votre établissement. Toute autre reproduction ou représentation, en tout ou partie, sous quelque forme et de quelque manière que ce soit, est interdite sauf accord préalable et écrit de l'éditeur, en dehors des cas prévus par la législation en vigueur en France. II est précisé que son stockage dans une base de données est également interdit. 


\title{
DE L'ALLEMAGNE : UN LIVRE SUR LA FRANCE
}

\author{
WINFRIED WEHLE*
}

Deux siècles après sa parution, l'essai de Germaine de Staël mérite une redécouverte. Bien que lissé par la civilité des salons, il fut à son époque un pamphlet. Avec le recul, l'excitation d'alors retombée, on peut apprécier son importance politico-culturelle. Manifeste entre l'Ancien Régime et les États nations, il tentait de lire les signes de l'ère nouvelle et posait les questions qui animent encore aujourd'hui le monde moderne. On n'est pas loin de penser que Madame de Staël, comme déjà dans De la littérature, s'efforce, dialoguant encore avec le Génie du christianisme de Chateaubriand, d'énoncer une nouvelle vision de l'avenir'. Elle reprend sous forme de l'essai la question laissée en suspens par son roman Corinne ou l'Italie : comment redéfinir une identité de l'individu et de la société dans les conditions d'une modernité postrévolutionnaire ${ }^{2}$. En 1810 , les besoins en étaient criants. Pour apprécier à sa juste valeur le diagnostic et l'intention de De l'Allemagne sur cette toile de fond, il faut un changement de regard provocateur. Car le titre est trompeur: De l'Allemagne est un livre sur la France ${ }^{3}$. La censure

* Universités de Bonn et d'Eichstaett

1. Son roman Delphine (1802) avait déjà anticipé une perspective développée maintenant par « De l'Allemagne » : «Il faudra [...] qu'un homme de génie s'enrichisse une fois par la féconde originalité de quelques écrivains allemands [...] et l'on pourrait parvenir à adapter au goût français, peut-être le plus pur de tous, des beautés originales qui donneraient à la littérature [i.e. française] du dix-neuvième siècle un caractère qui lui serait propre. » Voir Éd. L. Omacini / S. Balayé, Paris, Champion, 2004 (Textes de Litt. Mod. et Contemp. 75) ; Préf., p. 9.

2. Cf. ici l'essai complémentaire de l'auteur : «Trauma und Eruption : La littérature comme mise en scène de l'inconscient. Réflexions sur Corinne ou l'Italie de Madame de Staël ", in Revue d'Histoire littéraire de la France 1/2010, p. 35-64. ( http://edoc.ku-eichstaett.de/4245/).

3. Sur les controverses qu'avait provoquées ce livre, cf. le bilan d'A. Monchoux L'Allemagne devant les lettres françaises de 1814 à 1835, Toulouse (Fournié) 1953, p. 9-22, tout en soulignant qu'à la France est accordé le principale rôle (21), et il ajoute : "Madame de Staël met en relief ce qui l'intéresse et ne fait pas un compte-rendu objectif » (p. 16).

RHLF, 2014, $n^{\circ} 2$, p. 317-333 
napoléonienne l'a compris qui d'abord a supprimé sa conclusion émue dans le chapitre final « De l'influence de l'enthousiasme sur le bonheur ». On y lit : « Ô France ! terre de gloire et d'amour! si l'enthousiasme un jour s'éteignait sur votre sol, si le calcul disposait de tout, et que le raisonnement seul inspirât même le mépris des périls, à quoi vous serviraient votre beau ciel, vos esprits si brillants, votre nature si féconde ? Une intelligence active, une impétuosité savante vous rendraient les maîtres du monde ; mais vous n'y laisseriez que la trace des torrents de sable, terribles comme les flots, arides comme le désert ${ }^{4}$ ! »

Toute la force explosive du moment s'exprime, bien dosée, dans cette déclaration finale. Sous forme de concepts, elle reprend au fond une polarité anthropologique dramatisée dans les personnages de Corinne, Lord Nelvil et Lucile. Le nouveau livre est ainsi un rapport sur un voyage à l'intérieur du mal du siècle. Il a par conséquent, comme tant de témoignages de cette époque, des origines pathogènes. La déclaration d'amour préoccupée de l'auteur au pays qui fut sa patrie aussi bien intellectuelle que sociale, part certes du principe qui l'a aidé à acquérir la puissance et la gloire de l'Ancien Régime : la monarchie de la raison (Schiller) - calcul, raisonnement, intelligence, savoir, mais dans le seul but de lui ouvrir les yeux sur ses côtés destructeurs (désert) qui venaient de se manifester dans son pouvoir absolu. Les images (torrents de sable; terribles comme les flots arides) évoquent âprement un état de culture que la «Nouvelle science » de Giambattista Vico avait dépeint comme une barbarie funeste à l'horizon de l'Histoires Implicitement, la vision du monde de l'Ancien Régime a définitivement perdu son droit à l'existence. Plus aucun avenir heureux n'est promis en son nom. En ce sens, Mme de Staël prononce une critique fondamentale du rationalisme. Les censeurs de Napoléon - voire lui-même ? - l'ont étonnamment bien compris, qui se sont efforcés de museler son livre.

Le diagnostic reprend sous forme politico-morale l'interrogation sentimentale restée sans réponse dans Corinne ou l'Italie : comment conserver à l'avenir la grandeur de ce pays et l'amour pour lui ? La conclusion est à la fois un concentré programmatique de son voyage en Allemagne et une réponse à ce problème. Pour continuer à subsister en tant que « grande nation », la France doit se régénérer par l'anti-raison de l'enthousiasme. Comme la raison, l'enthousiasme avait parfaitement revendiqué ses droits dans l'anthropologie des Lumières. L'article de l'Encyclopédie en est

4. Cité d'après l'édition : Madame de Staël : De l'Allemagne (2 vol.), éd. S. Balayé, Paris, 1968 (GF 166/167), t. II, p. 316 (par la suite, abrégé avec mention du volume et du numéro de page).

5. Dont elle rejette cependant le modèle historique cyclique au nom d'une perfectibilité progressive de la civilisation (II, 229). 
l'impressionnant témoignage philosophique ${ }^{6}$. Le recours de Mme de Staël oriente cependant la discussion ${ }^{7}$ historique vers la modernité en ce sens que la lectrice de Rousseau comprend rationalité et enthousiasme comme une union contradictoire. L'un doit en quelque sorte socialiser ses penchants dictatoriaux dans le miroir de l'autre. En principe, elle songe déjà à une anthropologie dialectique. Mais, pour y parvenir, l'enthousiasme à la française doit être libéré de sa mise sous tutelle rationnelle. Le pays d'outreRhin est à même de montrer la créativité culturelle qui peut en résulter. La vie spirituelle de l'Allemagne est pour Mme de Staël une métonymie pour les bienfaits de cet enthousiasme. Elle oppose à la puissance de la raison les bienfaits de l'imagination (II, 313). Au sens le plus large, c'est l'ordre conflictuel à l'arrière-plan de cette campagne anthropologico-culturelle. Au nom de son appartenance spirituelle à la France, elle se sent amenée à exercer une critique envers la raison qui y aurait pris le dessus. Sa sympathie évidente n'est-elle donc pas en même temps le mode de lecture souhaité pour cet écrit?

L'impulsion directe lui a été fournie par le grand bouleversement historique de la Révolution française. Elle forme le trauma productif sur lequel elle travaille depuis son essai Des circonstances actuelles qui peuvent terminer la Révolution et des principes qui doivent fonder la République (1798) ${ }^{8}$. L'œuvre inachevée a trouvé dans De la littérature (1800) une suite à laquelle avait intensément réagi le Génie du christianisme (1802) de Chateaubriand, qui a largement obscurci son caractère pionnier ${ }^{9}$. Comme eux, Ch. Nodier (Moi-même), Senancour (Oberman), P.-S. Ballanche (L'Homme sans nom $)^{10}$ et d'autres ont vu dans la Révolution la fin du monde dont ils étaient issus. Ils font leur ce qui avait été consigné dans Des circonstances actuelles et qui accompagne plus ou moins chacun de leurs écrits comme une note dominante : « Hélas, nous souffrons tous. [...] L'avenir n'a point de précurseur. Le guide de la vraisemblance, de la probabilité n'existe plus. L'homme erre dans la vie comme un être lancé dans un élément étranger. Ses habitudes, ses sentiments, ses espérances, tout est confondu » (p. 2).

6. Cf. Encyclopédie ou Dictionnaire raisonné des Sciences, des Arts et des Métiers, réed. Stuttgart/Bad Cannstatt : Fromman/Holzboog, 1966, t. V, p. 719-722.

7. Reconstruite avec appareil critique par P. Geyer : Die Entdeckung des modernen Subjekts. Anthropologie von Descartes bis Rousseau; Tübingen , Niemeyer, 1997 (mimesis 29), en part. chap. 9, p. $200 s q$.

8. Éd. L. Omacini, Genève, Droz, 1979 (Textes litt. frç. 269).

9. Cf. G. Gengembre/J. Goldzink : «Introduction »; in Madame de Staël, De la Littérature, éd. les mêmes, Paris, 1991 (GF 629), p. 7-47 (pagination de cette édition entre parenthèses). Voir aussi l'appréciation de B. Steinwachs : Epochenbewusstsein und Kunsterfahrung. Studien zur geschichtsphilosophischen Ästhetik an der Wende vom 18. zum 19. Jh., Munich, Fink, 1986 (Theorie und Geschichte der Litt. und d. Schönen Künste 66), p. 62-86.

10. In Euvres complètes (1833), réimpression Genève (Slatkine) 1967, p. 251-288. 
Le début de De la littérature reprend cette 'ruine', de façon certes analytique mais avec une réflexion beaucoup plus avancée sur ce que l'on est en droit de nommer l'un de ses objectifs vitaux : " comment terminer la révolution ». De la littérature avait risqué une première réponse influente : « [montrer] la puissance que peut exercer la littérature sur la destinée de l'homme » (86). La littérature, les arts en général, sont pour le moins déjà reconnus comme les médiums privilégiés où la destinée postrévolutionnaire de l'homme se laisse traiter d'une manière adéquate. Mais les arts ne prennent-ils pas ainsi la place des divinités à qui l'on accordait jusqu'alors toute puissance sur nous - c'est-à-dire la raison paternelle et la nature maternelle ? On entrevoit ici ce que la révolution romantique des arts intégrera dans son programme fondamental comme l'engagement historique d'une première modernité. De l'Allemagne va plus loin en montrant dans l'image assombrie d'un présent révolutionnaire l'émergence d'un projet d'avenir philosophico-culturel.

Les nombreuses notes que Mme de Staël avait rassemblées au cours de ses voyages en Allemagne avaient fourni les bases thématiques. La forme d'écriture finalement retenue participe de trois traditions discursives de l'histoire culturelle et se justifie par le caractère hétérogène de l'Allemagne (I, 55). Ce faisant, elle anticipe le futur principe de mélange des styles sans toutefois le porter déjà en tant que tel au crédit de la modernité. D'une part, elle reprend, comme déjà dans son livre sur la littérature, le modèle de la «Querelle des Anciens et des Modernes » : la nouvelle école (II, 74) en Allemagne lui semble moderniste en cette tendance ; dans sa littérature et sa pensée, la France révolutionnairement bouleversée reste bien en deçà de ses nouvelles idées politiques. D'autre part, elle souscrit au discours ethnographique de «l'Histoire des civilisations » à la manière d'un Condorcet, qui tente d'expliquer comparativement les formes de vie, le climat, les mœurs, les coutumes, la langue et les arts. Ces deux points se sont fondés dans la perspective du genre des Parallèles. Non seulement Condorcet, mais aussi Montesquieu, ainsi que l'un de ses nombreux amis de corps et de l'esprit, August Wilhelm Schlegel ${ }^{11}$, l'ont mise en œuvre pour établir des identités à la lumière des altérités.

Le vagabondage de son regard forme un écho des étapes du voyage. Dans le déroulement de l'essai, les jugements obéissent à un schéma pertinent. Mme de Staël évalue toujours la culture des deux pays à l'aune du pour et du contre, elle les oppose dialectiquement : « On pourrait dire avec raison

11. Voir A.W. Schlegel, Parallèle entre la Phèdre d'Euripide et celle de Racine (Paris, 1807), avec une critique provocante du classicisme français. Il conclut par une citation - diplomatique du contemporain Laharpe : « Racine a partout substitué les plus grandes beautés [i. e. des anciens] aux plus grands défauts », in A.W. Schlegel, Euvres écrites en Français, éd. E. Böcking, rééd. Hildesheim, Olms, 1972 : Sämtliche Schriften, t. XIV, p. 405. 
que les Français et les Allemands sont aux deux extrémités de la chaîne morale ; [...] il n'en est point de plus opposées [i.e. des nations] dans leur système littéraire et philosophique » (I, 47). On voit apparaître un système général de rapports entre les deux nations : une partialité complémentaire. L'une est à même de pouvoir signaler à l'autre ce qui lui manque. À vrai dire, ce bilan déficitaire de la civilisation de l'un et de l'autre finit régulièrement par l'amener à se demander ce qui manque à sa propre patrie spirituelle. En un mot, celle-ci est jugée sur le point de perdre son prestige de phare culturel qu'elle a imposé à l'Europe au XVII ${ }^{\mathrm{e}}$ et XVIII ${ }^{\mathrm{e}}$ siècle. C'est de ce leadership intellectuel - et culturel - qu'elle est préoccupée. Il s'agit de le « renouveler », d'enrichir l'esprit qui le porte (I, $47 s q$.$) afin de régéné-$ rer dans son ensemble - à partir de la France - « la gloire des travaux de l'esprit humain » $(\mathrm{I}, 41)$ en général. Quel hommage honorifique à ce pays ! Tout doit partir de la question des causes de ce déclin. Elle reprend la position frontale des écrits précédents : « j’y manifestais les mêmes opinions et [...] j'y gardais le même silence sur le gouvernement actuel des Français » (I, 37), pour rendre d'autant plus attentif aux allusions «silencieuses ». La raison déterminante réside dans l'épuisement interne de l'Ancien Régime : « Le parti royaliste est entièrement et pour jamais fini et il ne lui reste qu'une puissance, celle de noyer avec lui tous ceux qui veulent se servir de lui comme parti pour un but quelconque »(Des Circonstances, 318/319). Ce jugement républicain est repris dans De l'Allemagne: « de nos jours, les hommes de la première classe n'ont aucune fonction dans l'état et n'étudient profondément aucune science, toute l'activité de leur esprit, qui devrait être employée dans le cercle des affaires ou des travaux intellectuels, se dirige sur l'observation des manières et la connaissance des anecdotes » (II, 230).

Si sa critique est aussi intransigeante, c'est qu'elle voit menacé le socle sur lequel elle se trouve elle-même, l'esprit de la société (I, 247), l'éthique de l'honnêteté, enrichie par la sincérité et le savoir. La loi du devoir (II, 301) est fondée sur lui. Cet idéal vertueux a perdu sa compétence à la fin du $\mathrm{XVIII}^{\mathrm{e}}$ siècle. Ne sont restées que ses perversions : superficialité maniérée (II, 301), calcul immoral (II, 302), frivolité, moquerie, plaisanterie, (I, 95 ; 106), conversation creuse, bref : la transformation de la précédente raison sociale en une raison égoïste (II, 301). Celle-ci s'épuise en raisonnements (II, 116) vaniteux. Le fondement esthétique de cet idéal social, le bon goût (I, 48 ; 247 et autres), s'en voit pareillement mis hors d'usage. Il n'est pas difficile de voir ici une déclaration politique à peine voilée pour la « perte d'ordre » actuel de la France : « C'est ainsi que la raison humaine s'habitue à la servitude » (I, 48). Mme de Staël ne cite aucun nom. Mais tout bon lecteur savait à qui elle faisait allusion.

Derrière la dictature, on voit se profiler ici, en grande ombre historique, la Révolution française elle-même, qui a dévoré ses enfants : «liberté ", 
" égalité », « fraternité ». Quand elle pouvait écrire encore plus librement comme dans De la littérature, elle l'a déclaré plus ouvertement : « La théorie philosophique de la Révolution de France est en elle-même incontestable; il n'y a de remède à ses effroyables abus qu'en jetant des torrents de lumière sur les principes et sur leur application » (25). Fait défaut la pratique de la lumière révolutionnaire par ses principes issus du siècle des Lumières. C'est notamment cette divergence entre idéal et réalité qui est à l'origine de ce "mal du siècle » qui s'exprima dans des regards préromantiques portés sur l'abîme. À l'instar du René de Chateaubriand, Corinne ne parvenait pas à se détacher de cette image au bord du Vésuve. Dans le chapitre « De la morale fondée sur l'intérêt personnel »(II, 181), De L'Allemagne en laisse entrevoir les raisons de façon plus analytique. Aux yeux de Mme de Staël, la Révolution, défigurée par la Terreur et par Napoléon, a trahi le modèle historico-culturel où son mouvement anarchique aurait pu être canalisé idéalement : «le perfectionnement [moral] comme destination de l'homme sur cette terre » (II, 196 ; 91). Au lieu de cela, le déchaînement des passions a radicalisé l'intérêt personnel. Ainsi, les hommes de bonne volonté n'ont pas été les seuls à être dévoyés. Des profiteurs s'en sont servis comme stratégie blâmable par intérêt personnel : « D'autres se sont proposés d'en abuser, et ils n'y ont que trop bien réussi » (ibid.). Les noms ne sont toujours pas cités. Mais toute personne de raison savait une fois de plus de qui il s'agissait pars pro toto. Le sens civique (élitaire) prêt à sacrifier à l'intérêt général (I, 190) était ébranlé dans sa pensée de perfectibilité. La critique vaut pour les personnages en vue comme pour les gouvernements.

Comment refermer ce gouffre qui sépare projet et résultat de la Révolution? Mme de Staël répond à la question en jetant un regard déroutant sur une Allemagne étrangère et sa vie de l'esprit pratiquement inconnue. Non pas que ce pays fût la France meilleure. La perspective de la voyageuse reste pour ainsi dire systématiquement fidèle à son intention fondamentale : tout pour la plus haute gloire de la France ! Elle recourt à une sorte de palinodie renversée. Toute estimation de la culture allemande est généralement précédée par une dépréciation de ses conditions politiques, sociétales et morales. La conclusion de l'ouvrage rejoint son entrée en matière : celui qui rencontre l'Allemagne en venant de France tombe sur une « civilisation encore nouvelle » (I, 57), qui reste à sa manière en devenir. Selon une théorie des climats répandue, le sombre paysage forestier du nord traduit bien - et il en est aussi la cause - le sentiment allemand de tristesse qui frappe le visiteur venant de la clarté du sud. Tout parle de travail, rien de plaisir. Une impression pénible $(\mathrm{I}, 52)$ plane sur l'ensemble. Le pays entier donne l'image qu'un grand peuple l'a déserté depuis longtemps! Les gens ressemblent au pays, privés de tout ce qui est en mesure de créer le caractère public : pas de religion commune, pas de gouvernement unifié, diversité de climats, 
aucune cohésion sociale. Celui qui prend comme norme un État fortement centralisé verra dans la mosaïque d'États allemands une paisible anarchie (II, 286). Elle n'est unifiée ni par une capitale ni par une société au sens français de l'esprit de société (I, 55). L'intérêt se perd dans un « chacun pour soi » individualiste (hommes privés ; II, 177), sans conscience nationale qui aurait pu faire naître un esprit public (ibid.). La manière allemande souffre d'un grave déficit, mesurée aux valeurs d'unité qui ont fait la grandeur de la France. Celle-là donne à voir ce que celle-ci va sacrifier si elle ne renouvelle pas les principes sociaux, philosophiques et esthétiques qu'elle a abandonnés dans la Révolution. Mme de Staël relativise en permanence son image de l'Allemagne. C'est un appel négatif à la France lui demandant de se rappeler son potentiel supérieur.

Cette « non-nation allemande » peut alors revendiquer la réciproque de sa maigre structure publique : en un mot, son « imaginatio borealis ». On voit apparaître des jugements qui ont connu longtemps un grand succès en tant que préjugés stéréotypés. L'image mentale esquissée par Mme de Staël est construite à l'inverse de celle de la France et porte ainsi l'accent sur une cohérence de la culture allemande qui se conforme cependant en bonne partie à son propre modèle culturel apporté : Nord contre Sud ; imagination contre réflexion; vie introvertie et privée (II, 177) contre vie extrovertie et publique $^{12}$. De ce rapport contrasté ressort finalement le point de référence suprême auquel elle soumet ses jugements : l'esprit de société français. Certes, elle privilégie les esprits supérieurs ; elle se montre ainsi élitaire, mais elle ne le fait plus dans le sens d'un état de classes hiérarchisé. Elle laisse, il est vrai, à l'idéal vertueux de «l'honnêteté » sa face cultivée, le « plaire ». Mais, dans le fond, elle voit la bienséance courtoise en quelque sorte intériorisée : « placer l'empire de nous en nous-mêmes » (II, 199). Vu sous cet angle, elle rejoint la mise en avant du sujet autonome, proclamée notamment dans le Plus vieux programme de système de l'idéalisme allemand, comme la pensée-modèle de l'homme des temps modernes ${ }^{13}$.

Ainsi, elle est sans doute à la portée de tout le monde. Et dépasse donc le cadre d'une société des happy few : chacun sera en mesure de la développer à partir de lui-même. Il s'agit là d'un tournant anthropologique de premier ordre qui changera sensiblement le fondement de la morale puisque cela signifie la fin d'un art de la société fondé sur la civilité élitaire. Celui qui, dans le passé, voulait se socialiser en son sens comme " honnête homme » devait satisfaire à un codex de classe bien défini. Mais un « perfectionnement »

12. C'est cette perspective que poursuit, à la suite de J. Starobinski, P. Winterling : Rückzug aus der Revolution. Eine Untersuchung zum Deutschlandbild und zur Literaturtheorie bei Mme de Staël und Charles de Villers, Rheinfelden, Schäuble, 1983.

13. «Das älteste Systemprogramm des deutschen Idealismus ", in Mythologie der Vernunft, éd. Chr.Jamme / H. Schneider, Frankfurt/M., Suhrkamp (stw 413) 1984 ; appendice p. 11-16. 
qui réside en nous-même, pose, il est vrai, lui aussi des limites à un libre épanouissement de notre subjectivité : il reste toujours relié à un impératif des Lumières. En tant que tel, cependant, il concerne en principe déjà le genre humain en général. Il n'est plus prescriptif, mais doit être conçu comme un vaste processus de formation s'étendant sur les siècles futurs, sous le signe de la justice (« la suprême loi, c'est la justice »; II, 188). Mme de Staël n'abandonne pourtant pas la foi, dont dépendent la vertu et le vice, par rapport à « une représentation de moi-même, en tant qu'être absolument libre $»^{14}$ comme on préconisait en Allemagne à l'époque ; elle la rattache, au contraire, au grand mouvement émancipateur du progrès.

Ce mouvement trouve un point de départ en Allemagne avec Leibniz (II, 120 sq.) en tant que défenseur éclairé d'une philosophie idéaliste. Celle-ci a toutefois son prix. Son pré-requis - l'individualisation des esprits - leur montre le chemin vers l'intérieur, justement dans l'introversion : « Il [i.e. l'homme] tend toujours plus en général à se replier sur lui-même [...] dans le plus intime de son être » (II, 43). Là, le contrôle exercé par la raison sociale s'efface pour favoriser l'esprit de réflexion (II, 80) et, ainsi, faire arriver au pouvoir sa faculté de l'âme : l'imagination, le mode de penser en images. Mais celle-ci se s'enfuit justement sur des territoires théoriques que de passer en actions pratiques (II, 80), même sur le plan militaire. Dans sa forme suprême, elle enfante la métaphysique : «Les Allemands voudraient arriver sur tous les sujets à des théories complètes, et toujours indépendantes des circonstances » (II, 69). Ce chemin de pensée ne mène pas à la clarté de la raison mais dans les profondeurs insondables de la spéculation. Il y règne l'obscurité de l'infini que l'on ne peut même plus rejoindre intelligiblement. Dans les souterrains de l'imagination se répand une spiritualité de l'âme (II, 120) qui, vue par la raison, est dépourvue de règles (II, 42), n'a aucun but raisonnable de connaissance (II, 16), mais tend surtout à un déchaînement anarchique (sans frein) de la pensée. En évoquant la vie de l'esprit allemande, Mme de Staël identifie ici, avec toute la réserve requise, un trauma qu'elle s'était déjà dépeint dans Corinne ou l'Italie : dans les tréfonds de l'être humain réside une énergie naturelle - qui sera plus tard scientifiquement identifiée comme l'inconscient. Elle se sent pour cela tenue de fixer des limites décisives à la manière allemande : « Il y a trop de métaphysique » (II, 89). Celle-ci mène pour finir à l'irrationalité, au fanatisme, suscite un délire de fièvre (II, 53), en dépit de toutes les systématisations érudites (II, 179). Au lieu de promouvoir le bien général, elle tend à un esprit de secte $(\mathrm{I}, 150 ; 286)$. L'Allemagne est pareillement clivée en tant que pays et nation. Se fait jour finalement la profonde inquiétude dans l'admiration pour les Allemands : une force créaturelle incontrôlée sous-tend toutes les spéculations de haut-vol. Leurs productions philosophiques et 
littéraires ne sont rien moins qu'en lien avec une révolution spirituelle (II, 256) : «C'était chez les Allemands qu'une révolution opérée par les idées devait avoir lieu » (II, 243). Pour les mêmes motifs que ceux que Mme de Staël porte au compte des excès de la Révolution française, elle tente aussi de protéger ses lecteurs d'une révolution allemande. L'une des intentions les plus signifiantes de De L'Allemagne est d'avertir les lecteurs des deux côtés de se garder d'une révolution.

Les risques quasi idéologiques du présent d'alors sont ainsi désignés : évacuation idéelle de la « raison » du côté français et décloisonnement idéel du côté allemand. Les conditions sont maintenant données pour reprendre la poignante question socio-historique : 'comment terminer la Révolution'? Depuis qu'elle a été posée dans les Circonstances actuelles (1798), la réponse mène à une totale création nouvelle (74). C'est aussi le but visé de l'intérêt supérieur de cet ouvrage sur l'Allemagne. Pour finir, La voyageuse offre ici une vision vers la modernité. Celle-ci atteint son apogée dans la conclusion portée au pathétique. Un trait de caractère propre aux Allemands échappe ostensiblement à ses réserves critiques : l'enthousiasme. C'est grâce à lui que la nature française doit recouvrer la santé. Dès le début déjà, dans son essai De l'influence des passions sur le bonheur des individus et des nations daté de 1796, elle avait recherché ce fondement ${ }^{15}$. Elle n'avait alors pas vu d'autre issue que l'ataraxie des héros des tragédies classiques : l'indépendance morale de l'homme, obtenue en s'abstenant de toute passion et en ignorant les affects des autres (75/76). Mais une vie dans la tourmente peutelle être apaisée dans ce cimetière des sentiments ? Maintenant, dans De l'Allemagne, elle a trouvé l'approche pour reprendre la question de façon constructive : ce sera dans le cadre d'une anthropologie de la morale philosophique. Kant n'est pas le moindre de ses témoins. Une argumentation rigoureuse interdit tout cheminement de pensée impulsif. En tout cas, les bases sont évidentes. Elles confèrent au livre le rang d'une œuvre de transition entre l'Ancien Régime et la modernité postrévolutionnaire.

Avec autant d'emphase que de respect, Madame de Staël désigne désormais l'enthousiasme comme ressource incontournable de la connaissance humaine. Celui qui y puise est inspiré divinement, tout à fait en écho du pneuma antique. Au sens littéraire plus étroit, elle pouvait se référer aux leçons de l'inspiration (II, 307). La revalorisation de l'enthousiasme de la période des Lumières s'y est indéniablement investie dans l'Encyclopédie ${ }^{16}$.

15. Essai sur les fictions suivi De l'Influence des Passions, éd. M. Tournier, Paris, Ramsay, 1979, p. $53 s q$.

16. Là, sous la protection de la « raison », il pouvait se sentir protégé contre tous les soupçons traditionnels : « c'est la raison seule [...] qui le fait naître [...]. Il est, si on ose le dire, le chef-d'œuvre de la raison » (Denis Diderot, Euvres complètes, t. V, éd. P.J. Lough et J. Proust, Paris 1976, p. 121). D'ailleurs, sa production est déjà réservée au seul génie et aux arts (ibid., p. 121). 
Toutefois, pareil à un renversement intellectuel, il doit maintenant prendre en main la direction des choses humaine - et remettre à sa place la raison (entendement) historique. En tant que disposition de l'âme (II, 303), il est promu comme bagage humain fondamental précédant toute formation sociétale et cognitive. Cela ne vaut-il pas aussi pour le « point suprême » auquel André Breton voulait soumettre une théorie de la connaissance surréaliste ? Il est d'une part une faculté de l'âme, mais d'autre part aussi un don naturel imprescriptible. D'une manière ou d'une autre, il correspond à un « élan vital » universel (II, 301), naturellement créatif, qui pourvoit tout un chacun en énergie ${ }^{17}$, qu'il soit de noble naissance ou de basse extraction. Madame de Staël pense avoir trouvé là le point archimédique pour pouvoir contrer le profond bouleversement d'une conception de vie rationaliste et l'établir d'une façon nouvelle. Elle propose cet enthousiasme comme contrepoids (contrebalance; II, 302) d'un calcul fanatique et égoïste. Mais s'il s'agit bien là d'une universalité humaine, comment expliquer alors l'effondrement de la Révolution française ? Pour autant qu'un sentiment enthousiaste puisse être impérativement entraînant, il faut, afin d'éviter les risques de ses débordements observables à l'exemple de l'Allemagne, le cultiver et le socialiser, autrement dit le canaliser par le contrôle de la raison, son opposé anthropologique. Les forces de la raison doivent ainsi concilier les bouillonnantes réflexions spéculatives et l'intérêt général. C'est sur leur échange que l'homme est élémentairement établi en tant que double nature : «Ce sentiment [i.e. de l'enthousiasme] est par son universalité même très favorable à la pensée et à l'imagination » (II, 306). Certes, Mme de Staël n'en a pas autrement systématisé les conséquences. Mais elle est de facto sur le point de réinterpréter fondamentalement l'arbre de la connaissance des Lumières. Celui-ci avait pratiquement mis sur le même plan les trois facultés anthropologiques traditionnelles de l'homme - pensée, sentiment, volonté - parce qu'il s'enracinait dans un entendement supérieur qui tiendrait en échec les excroissances de leurs intérêts particuliers ${ }^{18}$.

ENTENDEMENT

$\begin{array}{lcc}\text { Mémóre } & \text { Raison } & \text { Imagination } \\ \text { Histoire } & \text { Philosophie } & \text { Poésie } \\ \text { lté de sensation] } & \text { [Faculté de compréhension ] } & \text { [Faculté de désir] }\end{array}$

17. Voir à ce sujet l'étude de base de M. Delon : L'idée d'énergie au tournant des Lumières, Paris, Puf, 1985.

18. D. Diderot, Euvres complètes, op. cit. p. 121. 
La Terreur avait cependant prouvé que la raison elle-même n'était pas en mesure de garder raison ${ }^{19}$. Mme de Staël a reconsidéré les conditions anthropologiques de la connaissance et de la morale. Nos actions sont vraies et bonnes quand les passions, moteur vitaliste de la pensée et de l'action ${ }^{20}$, sont négociées dans un dialogue entre raison et imagination et soumises à un bon sens humain. Elles constituent aussi bien la force motrice pour la formation d'un esprit de société - principe sociétal - que pour l'inspiration du génie (II, 306-7) - principe créatif. Que la sympathie aille plutôt à l'imagination est l'une des requêtes principales de De l'Allemagne : " L'esprit est d'une moins illustre origine; il sert à contester, mais le génie est créateur, parce que son enthousiasme génère en lui un sentiment de l'infini» (II, 241).

Ce n'est qu'ainsi que l'État et la société retrouveraient aussi leur ordre précédent après la destruction révolutionnaire et qu'ils satisferaient à l'impératif de "liberté ». "L'entendement» des Lumières a perdu en tout cas sa fonction de souverain. Où et comment se laisserait alors envisager, pour reprendre la formule future de Victor Hugo, une « harmonie des contraires $»^{21}$ ? Autrement dit : il faudrait une herméneutique culturelle permettant un nouveau tiers commun, une solide politique morale.

Si l'on en croit la profonde conviction de Mme de Staël dans tous ses écrits, la lumière peut revenir dans un présent assombri à condition que les Lumières inachevées soient encore pratiquées avec encore plus de lumière. De l'Allemagne n'approfondit en ce sens qu'une argumentation déjà développée dans De la littérature : "Quel siècle s'ouvrira pour nous, si la raison $[\ldots]$ prend pour guide la raison $![\ldots]$ il n'y a de remèdes à ces effroyables abus [i.e. de la révolution] qu'en jetant des torrents de lumière sur les principes et sur leur application » (25). Ainsi, l'entendement et la raison resteraient-ils tout de même les garants d'une pensée et d'une action humaine? L'éloge d'une imagination excitée par l'enthousiasme

19. Le compagnon de Mme de Staël, Benjamin Constant, activiste de la Révolution, avait publié en 1797 l'ouvrage Des Effets de la Terreur. On peut y lire, par exemple : « Les hommes ou les corps revêtus de pouvoirs sans bornes [allusion à la loi de Robespierre pour le tribunal révolutionnaire du 10.06.1794], deviennent ivres de ces pouvoirs. Il ne faut jamais supporter que, dans aucune circonstance, une puissance illimitée puisse être admissible, et dans la réalité jamais elle n'est nécessaire » (in Benjamin Constant, Euvres Complètes, t. I, éd. M. Barberis, Tübingen, Niemeyer, 1998 ; p. 519).

20. Dans « De l'Influence des Passions », il est écrit : « Les passions, cette force impulsive qui entraine l'homme indépendamment de sa volonté, voilà le véritable obstacle au bonheur individuel et politique » (p. 59). Ici, elles sont encore soumises à une stricte censure. Voir R. Rey, Naissance et développement du vitalisme en France, Oxford (Voltaire Foundation) 2000, p. 187 sq.

21. Lui-même trouvait - encore et toujours - refuge dans le concept du « goût » frappé au coin de la bonne société, mais désormais comme régulateur de l'imagination créatrice : « Le goût, c'est la raison du génie » (451) ! Toutefois, il le relève déjà de son lien avec le « beau » classique et le remplace par le «caractéristique » (437). Cf. la «Préface de Cromwell», in V. H., Théâtre complet, t. I, éd. J.-J. Thierry/ J. Mélèse, Paris, 1963 (Pléiade), p. 409 sq. 
n'était-elle finalement que l'inspiration d'un voyage dans l'altérité de l'Allemagne, qui, au retour, est de nouveau rappelée à l'ordre par le genius loci. D'où devrait alors venir la nouvelle « lumière »? Comment exercer la raison sans succomber à son penchant destructeur ? La solution consiste « à placer l'empire de nous en nous-mêmes » (II, 199). Nous développons des sentiments humains quand nous ne nous considérons plus comme objet exclusif de notre intérêt. En conséquence, notre raison doit littéralement se relativiser en se mettant au service de ce que les sensations et les impressions, bref, la nature profonde (âme) ont à lui dire : « Le calcul est l'ouvrier du génie, le serviteur de l'âme ; mais, s'il devient maître, il n'y a plus rien de grand ni de noble dans l'homme » (II, 183). On ne lui demande ici rien de moins que de mettre en scène la nature, son contreprincipe. C'est la nature et non pas la raison qui a accès à la « source noble » de la morale (II, 200) et de la philosophie : « Les vraies causes finales de la nature ce sont ses rapports avec notre âme et avec notre sort immortel » (II, 300). Par essence, la nature est bonne pour l'homme dans la mesure où il fait naturellement un avec elle, comme Mme de Staël l'argumente avec Novalis (II, $292 s q$.) Elle peut en retirer de nouveau un principe optimiste bien que Rousseau eût vainement tenté de le confirmer. L'animalité que Hobbes ou le marquis de Sade avaient appris à craindre dans le principe vital (II, 294) de la nature se laisse en quelque sorte amalgamer c'est son principe salvateur - quand le corps et l'esprit sont mis - génialement - en harmonie.

Qui serait mieux à même de réaliser cette entremise (rapports) que les arts ? « La poésie et les beaux-arts servent à développer dans l'homme ce bonheur d'illustre origine » (II, 310), i.e. l'harmonie universelle (II, 301). Dans leurs espaces de liberté esthétique, les antagonistes que sont la sensibilité et la raison peuvent, comme nulle part ailleurs, revêtir une forme langagière et picturale, sans contrainte d'action immédiate. Les arts forment pour ainsi dire un troisième lieu de connaissance entre celui qui s'intéresse à l'exigence de vérité et celui qui est dominé par les contraintes du désir. Tous les deux peuvent y mettre en scène leur conflit et faire jouer librement les possibilités d'une médiation au sens de «perfectibilité ». Mais cela requiert avant tout une conduite langagière incontestable. Mme de Staël fait valoir que c'est la littérature qui est le mieux à même de modérer ce dialogue anthropologique. La nature de l'esprit, qui s'accomplit dans l'acte de penser, produit des idées, conceptualise ce qu'elle saisit. La philosophie systématique en est la suprême forme culturelle. L'esprit de la nature s'exprime en revanche autrement : il se sert de l'imagination et parle conformément à elle, c'est-à-dire en « images », la poésie étant sa plus haute forme culturelle. Des progrès dans le processus de perfectibilité seraient alors - de nouveau - possibles, si chacun des deux 
langages consentait à un compromis et fixait ainsi des limites à sa propre force discursive tendant à la monomanie. Car « la pensée n'est rien sans l'enthousiasme » (II, 303). Et, inversement, une imagination envolée par l'enthousiasme ne serait rien sans ajustement cognitif, notamment là où il en va de la communauté tout entière : «Les nations libres ont besoin de l'un et de l'autre » (II, 303). Mme de Staël fait ici encore référence à son herméneutique de la culture aux accents modernes qui, par la suite, fera date en tant que dialectique de la modernité. Sur ce point précis, elle touche du doigt l'une des perspectives les plus audacieuses de l'idéalisme allemand comme, encore une fois, Le plus ancien programme systématique l'avait inscrite de façon prophétique dans le concept de la modernité : « Nous devons avoir une nouvelle mythologie [qui parle à notre sens esthétique], mais cette mythologie doit se trouver au service des idées. [...] La poésie en recevra une plus haute dignité, elle redeviendra à la fin ce qu'elle fut au commencement - l'éducatrice de l'humanité22. »

Là aussi la liberté est élevée en principe supérieur de l'action humaine. Toutefois, celui qui, comme Mme de Staël, avait sous les yeux les exactions commises au nom de la liberté devait avoir une approche morale différente de celle des idéalistes allemands. Elle se voit contrainte de placer doublement son concept de liberté dans l'éclairage des Lumières. À la vue du désordre révolutionnaire, elle s'en tient à un idéal sociétal - révisé - du XVIII ${ }^{\mathrm{e}}$ siècle. La France a toujours créé le type même de modèle pour l'Europe, « où l'esprit de société régnait si éminemment » $(\mathrm{I}, 247)$. En dépit de tout épuisement frivole, stérile, conventionnel, le grand monde, la bonne compagnie, l'homme du monde (I, 247), le lieu social du salon, passent pour elle encore et toujours comme le prestigieux cadre structurant d'un futur projet de société, dans lequel le caractère mondain de l'ancienne époque et celui, égalitaire, de la nouvelle fusionneront en une classe dirigeante postrévolutionnaire d'hommes supérieurs (I, 162). Sans l'exprimer ouvertement, Mme de Staël a érigé en exemple général le style de vie libéral de son domaine familial à Coppet. Il est toujours autant marqué par un critère auquel se fiait déjà le XVIII ${ }^{\mathrm{e}}$ siècle classique éclairé : le bon goût (I, chap. XIV; $247 s q$. . $^{23}$ : « Le goût est en littérature comme le bon ton en société » (I, 247). En société comme dans les Beaux-Arts, il est garant de tempérance classique : contrôle des ambitions (sociales) de

22. Op. cit., p. 13.

23. Déjà établi dans De la Littérature comme fondement de sa critique du présent (op. cit., t. II, chap. 2 ; p. 297 sq.). Dans ce présent d'alors, le principal défenseur d'un correctif classique à apporter à la trahison des théories éclairées de la Révolution fut cependant J. F. de La Harpe avec la parution de ses cours réputés Lycée, ou Cours de Littérature ancienne et moderne, Paris 17891805 , en 24 volumes. D'après lui, le "goût » (joint à la monarchie et à la religion) forme le contrepoids à la barbarie de la Révolution. Cf. par exemple t. I, p. $45 s q$. 
l'amour-propre; modération de l'imagination débordante par la bienséance stylistique - toutes choses qui, à la fin du XVIII ${ }^{\mathrm{e}}$ siècle, s'étaient figées en mascarade. Pour pouvoir continuer à offrir le bon goût culturellement et esthétiquement, il faut pour ainsi dire, le re-naturer. Ce qu'il avait perdu, on le comprend bien à l'exemple de l'Allemagne. C'est l'enthousiasme qui est cultivé là-bas en tant que génie et qui bannit toute étroitesse d'esprit, tout cliché et toute platitude. Car le principe social du goût sans génie ne fait que se figer et fossiliser. En revanche, sans lien avec le «goût », le "génie », en tant qu'inspiration d'un individualiste solitaire, outrepasserait toute morale et tout caractère raisonnable et ne renoncerait même pas à la description du grotesque, à l'exemple des tragédies d'un Zacharias Werner ${ }^{24}$. Mme de Staël vise sans doute ici aussi les mélodrames et romans noirs contemporains qui fascinaient les masses ${ }^{25}$ et qui ont ouvert la voie à une esthétique du laid et du diabolique.

On touche ici à l'une de ses plus profondes inquiétudes : la perte d'une pièce maîtresse dans sa vision du monde fondée sur le $b e a u^{26}$. En son nom, les facultés de pensée et d'imagination semblaient jusqu'alors pouvoir accepter une médiation harmonieuse. «Retrouver la source des grandes beautés » $(\mathrm{I}, 48)$ se trouve déjà, telle une exhortation, dans la préface de $D e$ l'Allemagne. Et son analyse se conclura aussi par une réflexion à ce sujet. Si c'est l'enthousiasme qui peut éveiller en nous l'amour du beau, le sentiment de beauté est pour sa part inné, un don de la nature. Par son origine, il nous permet de "goûter toutes les merveilles du cœur et de la pensée ». Il revêt ainsi les traits d'une religiosité profane. En conséquence, il lui faut une liturgie symbolique appropriée ${ }^{27}$. La loi du devoir (II, 301) décide de son culte ; son exécution repose dans les mains de la poésie et [des] beaux arts qui se trouvent seuls à même - avec leurs institutions - de " développer dans l'homme ce bonheur d'illustre origine » (II, 310). Ils peuvent capter les aspirations élevées de l'imagination humaine, issues des profondeurs de

24. Cf. E. Wismer, Der Einfluß des deutsche Romantikers Zacharias Werner in Frankreich. Die Beziehungen des Dichters zu Mme de Staël, Bern, P. Lang, 1968.

25. Caractérisés déjà par des contemporains de façon à la fois ironique et réaliste ; voir « Le mélodrame aux Boulevards. Facétie littéraire, historique et dramatique par Placide » [i.e. Armand Charlemagne], Paris 1809. Voir aussi l'appréciation historico-culturelle de Charles Nodier, «Introduction » au Théâtre choisi de G. de Pixerécourt (4 vol.), Nancy, 1841-1843 ; réimpression Genève, Slatkine 1971, t. I, p. XLIV-LXXXVIII.

26. «Ce qui est vraiment divin dans le cœur de l'homme ne peut être défini ; s'il y a des mots pour quelques traits, il n'y en a point pour exprimer [...] le mystère de la véritable beauté dans tous les genres » (II, 205).

27. Cette vue des choses ne semble pas échapper à l'influence du culte révolutionnaire de " l'Être suprême ", dans lequel l'intellect et la nature devaient se réconcilier dans l'esprit de la République, misant par là même sur une universelle religiosité de l'art. Voir ici les descriptions, d'A. Aulard, «Le culte de la raison et le culte de l'être suprême » (Paris 1892), réimpression Aalen (Scientia) 1975, p. 103 sq. Le tableau de Pierre-Antoine Demachy nous en donne une représentation : «Fête de l'Être suprême au Champ-de-Mars » (8 juin 1807), Musée Carnavalet, Paris. 
l'âme (II, 310), en un art réfléchi, raisonné et apporter ainsi une sorte de preuve esthétique de l'existence de Dieu à l'image de l'homme. Ce n'est que par ce relais qu'ils pourraient prendre fait et cause pour les nobles concepts communs du vrai et du bon. Il en ressort que les arts sont à considérer comme l'institution morale de l'avenir, digne de l'éducation du genre humain ${ }^{28}$.

C'est ainsi que Mme de Staël tente de renouveler l'épistémologie classique des Lumières pour la sauvegarder au-delà de la Révolution. Quelles que soient les suggestions qu'elle emprunte à l'Allemagne, celles-ci servent à «nous [i.e. les Français] enrichir en les modifiant à notre manière " $(I, 48)$. Le génie ainsi cultivé ne peut s'épanouir qu'en France. En dépit de tous les signes de désagrégation révolutionnaires, la législation de bon goût et l'élégance de la société y sont toujours ancrées dans la conscience publique. Pour devenir une nation efficace et une société civilisée, l'Allemagne devrait donc s'en tenir à l'exemple de la France : «Les Allemands feraient bien de profiter, sous des rapports essentiels, [...] des avantages de l'esprit social en France : ils devraient apprendre des Français » (I, 110). L'ouvrage De l'Allemagne ne peut et ne veut pas cacher d'être voulu comme une sorte de «Défense et Illustration de la langue française » moderne malgré tout le respect pour l'altérité de l'Allemagne.

La révolution de Juillet jettera sur cet « esprit de société » élitaire une ombre profonde qu'il le fait passé et perdu. Saint-Simon et ses adeptes l'avaient déjà remplacé par une « fraternité » de toutes les classes faisant office de religion sociale ${ }^{29}$. Victor Cousin militait dès 1818 pour un partage des domaines épistémologiques ${ }^{30}$. Certes, il conservait encore - comme concession à une société restauratrice - le « goût » en tant que correctif (moral). Mais, en réalité, c'est la perte d'une totalité classique des Lumières

28. Il est difficile de savoir jusqu'où Mme de Staël avait déjà appréhendé et intégré dans ses pensées le projet éducatif schillérien d'un état esthétique. Dans « Les lettres sur l'éducation esthétique de l'homme », Schiller avait pourtant déjà milité en 1794 pour une révolution esthétique qui surpasse non seulement l'éducation naturelle comme celle développée par Rousseau dans Émile, mais aussi tout État de raison, aussi parfait soit-il, parce qu'elle est en mesure, dans son inclination au jeu que lui permet l'art, de transformer l'élément du beau dans la liberté de l'art en une expérience morale. Voir notamment les lettres XXI à XXVI.

29. Là on peut lire : «Toute la morale sera déduite directement de ce principe : Les hommes doivent se conduire en frères à l'égard les uns des autres; et ce principe, qui appartient au christianisme primitif, éprouvera une transfiguration d'après laquelle il sera présenté comme devant être aujourd'hui le but de tous les travaux religieux. Ce principe régénéré sera présenté de la manière suivante : la religion doit diriger la société vers le grand but de l'amélioration la plus rapide du sort de la classe la plus pauvre ». Voir « Naissance du Christianisme », in Euvres de Claude-Henri Simon, t. II, Paris, 1966 (rééd. de l'éd. V. E. Dentu, Paris, 1869, t. II, p. 174-187) ; ici p. 117.

30. Victor Cousin, Du Vrai, du Beau et du Bien, Paris, $1^{\text {re }}$ éd. 1818 ; huitième leçon, p. 174188. 
qui sous-tend son concept de différenciation des sphères de valeur ${ }^{31}:$ «Trois facultés entrent dans cette faculté complexe qui se nomme le goût : l'imagination, le sentiment, la raison. » Malgré toute l'estime qu'on puisse lui porter, le « goût » n'a cependant plus de compétence privilégiée pour l'art, qui, par sa liberté, pourrait lui servir plus que toutes les autres facultés : « [...] il n'en est pas moins vrai que l'art, l'État, la religion, sont des puissances qui ont chacune leur monde à part et leurs effets propres; elles se prêtent un concours mutuel; elles ne doivent point se mettre au service l'une de l'autre ${ }^{32}$. »

Certes, un bon nombre de réponses par lesquelles « De l'Allemagne » a réagi aux problèmes internes et externes de l'époque seront bientôt dépassées - tout comme Madame de Staël surmonta elle-même, avec son écrit de 1810, le dilemme sur lequel elle avait laissé son roman «Corinne ou l'Italie » de 1807. Corinne, incarnation d'une imagination et d'une improvisation proches de la nature, et Lord Nelvil, parangon du respect des convenances sociétales, ne trouvent aucun moyen de concilier leurs différences. Ils pressentaient toutefois, sans pouvoir l'adopter, un « troisième » point de vue capable de les lever : un art autoréférentiel, à la fois paradigme d'une vie autodéterminée. Mais cela aurait signifié d'une part abandonner "l'âme » ${ }^{33}$, séculaire concept unitaire, d'origine humoralpathologique de l'homme, dont elle avait d'autre part besoin pour prendre la liberté de louer le propre de la créativité humaine dans la nature créaturelle de l'homme. Significatives étaient ici aussi, dans « De l'Allemagne », les questions soulevées par cet écrit parce qu'elles n'ont plus cessé d'êtres posées depuis. Elles ont en cela suscité plus et autrement de modernité que Mme de Staël ne pouvait l'envisager au moment historique. Mais la place particulière qu'elle avait déjà attribuée à la poésie - en tant que projet dans Corinne ou l'Italie ou de façon programmatique dans son essai sur l'Allemagne - va ensuite, à l'apogée d'un romantisme français, chez un Lamartine par exemple, chercher refuge auprès de l'autonomie poétique qu'elle avait bien perçue en Allemagne mais rejetée comme révolutionnaire. Dans l'ouvrage de Lamartine Des destinées de la Poésie, publié en 1834, on lit : « Qu'est-ce, en effet, que la poésie ? [...] c'est la langue complète, la langue par excellence qui saisit l'homme par son humanité tout entière » [et pas seulement par le goût ]. D’où, par conséquent : «La première chose que fait maintenant l'esprit [!] du lecteur,

31. Au sens de M. Foucault, Les mots et les choses, Paris, 1966 ; t. I, chap.2 : « La prose du monde ».

32. Op. cit., p. 188.

33. « L'âme est un foyer qui rayonne dans tous les sens ; c'est dans ce foyer que consiste l'existence. » En cela, Mme de Staël garde encore un soutien anthropologique traditionnel à son concept subjectif (ce moi; II, 96). 
c'est de [...] chercher la poésie [i.e. la raison chantée] seule dans l'œuvre poétique $^{34}$. » L'art peut désormais revendiquer ouvertement et durablement son droit, être le premier dans la conduite des affaires humaines. L'identité qu'il offre a toutefois son prix moderne post-métaphysique. Il ne peut plus dire, ce que - substantiellement - c'est un moi ou doit être, mais comment il peut se forger son moi. C'est là le projet d'une anthropologie générative. Prométhée en est son parrain mythique ${ }^{35}:$ "Tout est progressif, tout marche » (I, 215).

34. In Euvres complètes de Lamartine, Paris, 1862, t. I, p. 25-62.

35. Pour la grande présence de celui-ci dans l'imaginaire de l'époque, voir par ex. Chateaubriand dans la stylisation de son panorama de l'époque, les Mémoires d'Outre-tombe : « Il y a là une sorte de vocation prométhéenne de l'homme ; [...] une sorte de volonté de conquête » (Paris, Librairie Générale Française 1973, p. 509). - Voir pour l'ensemble R. Trousson, Le Thème de Prométhée dans la littérature européenne (2 vol.), Genève, Droz, 1962 ; notamment t. II, p. 293 sq., avec toutefois peu de références au préromantisme français. 